\title{
Estimativa das perdas de solo por erosão hídrica utilizando o Método de Erosão Potencial
}

\section{Estimation of soil losses by water erosion using the Erosion Potential Method}

\author{
Guilherme Silva Pinto $\bowtie(\mathbb{D}$, Lucas Emanuel Servidoni $\varangle(\mathbb{D}$, Guilherme Henrique Expedito \\ Lense $\triangle$ (iD, Rodrigo Santos Moreira $\varangle$ (iD, Ronaldo Luiz Mincato* $\varangle$ (iD \\ Instituto de Ciência da Natureza, Universidade Federal de Alfenas-MG, \\ Recebido (Received): 24/07/2019 \\ Alfenas, Minas Gerais, Brasil \\ Aceito (Accepted):03/04/2020
}

E-mails: guilhermesilpin@hotmail.com (GSP); les.servidoni@gmail.com (LES);

guilhermeelense@gmail.com (GHEL); rodrigo.moreira@unifal-mg.edu.br (RSM)

*E-mail para correspondência: ronaldo.mincato@unifal-mg.edu.br

\begin{abstract}
Resumo: A erosão hídrica é a principal forma de degradação dos solos tropicais, gerando inúmeros prejuízos ambientais e socioeconômicos. As estimativas das taxas de perdas de solo por erosão hídrica são importantes para avaliar a degradação do solo e para proposição de medidas de manejo conservacionistas. Dentre os diversos modelos, o Método de Erosão Potencial se destaca pela facilidade de aplicação e baixo custo de implementação para estimar as taxas de perdas de solo. Dessa forma, o objetivo do trabalho foi aplicar o Método da Erosão Potencial para estimar as taxas de perdas de solo por erosão hídrica em uma sub-bacia hidrográfica do sul do Estado de Minas Gerais. A área estudada foi a sub-bacia hidrográfica do Córrego do Pântano II, no Município de Alfenas, sul de Minas Gerais, Sudeste do Brasil. O coeficiente de intensidade de erosão foi de 0,347 , que indica o predominio de erosão de fraca intensidade. A perda total de solo foi de $816,48 \mathrm{Mg} a n o^{-1}$, com perda média de $1,31 \mathrm{Mg} \mathrm{ha}^{-1} \mathrm{ano}^{-1}$. Conforme esperado, as áreas sem cobertura vegetal e com relevo íngreme apresentaram as maiores taxas de perda, com cerca de $1,0 \%$ da sub-bacia com perdas acima do limite de Tolerância de Perda de Solo. O Método de Erosão Potencial pode ser utilizado nas condições edafoclimáticas tropicais para apontar as áreas com maior ocorrência de erosão hídrica, sendo uma alternativa eficaz, simples e de baixo custo para identificar áreas prioritárias na proposição de ações para mitigação dos impactos ambientais associados ao fenômeno.
\end{abstract}

Palavras-Chaves: Geoprocessamento; Modelagem; Sensoriamento Remoto.

\begin{abstract}
Water erosion is the main form of degradation of tropical soils, generating numerous environmental and economic losses. Estimates of soil loss rates due to water erosion are important to assess soil degradation and propose conservation management measures. Among the various models, the Potential Erosion Method stands out for its ease of application and low cost of implementation to estimate soil loss rates. Thus, the objective of the work was to apply the Potential Erosion Method to estimate the rates of soil loss due to water erosion in a hydrographic subbasin in southern Minas Gerais state. The studied area was the hydrographic subbasin of the Córrego do Pântano II, in the Municipality of Alfenas, south of Minas Gerais, Southeastern Brazil. The erosion intensity coefficient was 0.347, which indicates the predominance of low intensity erosion. The total soil loss was $816.48 \mathrm{Mg} \mathrm{year}^{-1}$, with an estimated average of $1.31 \mathrm{Mg} \mathrm{ha}^{-1}$ year ${ }^{-1}$. As expected, areas without soil cover and with steep relief presented the higher soil loss rates, with about 1.0\% of the sub-basin above the Soil Loss Tolerance limit. The Erosion Potential Method can be used in tropical edaphoclimatic conditions to indicate the areas with the highest occurrence of water erosion, being an effective, simple and low cost alternative to identify priority areas in the proposal of actions to mitigate the environmental impacts associated with this phenomenon.
\end{abstract}

Keywords: Geoprocessing; Modeling; Remote Sensing. 


\section{Introdução}

A erosão hídrica é um fenômeno natural, que, em termos econômicos, é a principal forma de degradação dos solos tropicais, gerando vários prejuízos ambientais e socioeconômicos (DECHEN et al. 2015). Os danos causados pela erosão incluem tanto impactos locais, como danos às plantações, perda de capacidade de armazenamento de água, remoção de nutrientes, matéria orgânica e agroquímicos; como impactos fora do local onde os sedimentos são gerados, como assoreamento de corpos hídricos, poluição da água e danos à infraestrutura causados por inundações lamacentas (POSTHUMUS et al. 2015). A erosão hídrica é intensificada pelas atividades humanas, por alterações no uso da terra e práticas de manejo inadequadas como plantio em desnível, pastoreio exagerado, queimadas e trânsito excessivo de maquinário agrícola (PANDEY et al. 2016).

Globalmente, cerca de 75 bilhões de toneladas de solo são perdidas a cada ano nas terras cultivadas, devido à erosão hídrica, gerando um prejuízo de aproximadamente US \$ 400 bilhões (FAO, 2017). Na União Europeia, as perdas em produção agrícola causadas pela erosão são de cerca de $0,12 \%$ ao ano, o que se traduz em US \$ 333,09 milhões (PANAGOS et al. 2018). Quanto ao Brasil, são perdidas 847 milhões de $\mathrm{Mg}$ ou t por ano (MERTEN e MINELLA, 2013), com custos anuais de US \$ 1,65 bilhões, na cotação do dólar de 2002, relativos aos cultivos temporários e permanentes (HERNANI et al. 2002). A erosão hídrica reduz a disponibilidade de solos férteis e a qualidade da água, promove a degradação ambiental das terras cultivadas e compromete a sustentabilidade do sistema (SCHARRÓN e SÁNCHEZ, 2017). Além do transporte de sedimentos, causa perda de nutrientes, agroquímicos, contaminantes e matéria orgânica e gera custos adicionais aos produtores (AVANZI et al. 2013).

Nos últimos anos, em meio a preocupação com a conservação do solo e da água, foram desenvolvidos vários modelos para a estimativa da erosão hídrica (LOVRIC e TOSIC, 2018). Os modelos apresentam ampla praticidade e possibilitam a aplicação em diferentes usos de solo (AMORIM et al. 2010). Além disso, são vantajosos pela facilidade de execução quando comparados a métodos diretos que necessitam de experimentos em campo com coletas contínuas de dados, considerados trabalhosos e de custo elevado (BARROS et al. 2018).

Dentre os diversos modelos disponíveis, o Método de Erosão Potencial (EPM) (GAVRILOVIC, 1988) foi amplamente utilizado em locais de clima subtropical, na Europa Oriental, Oriente Médio e Norte da África (TAVARES et al. 2019). Por isso, recentemente foi adaptado às condições edafoclimáticas tropicais apresentando estimativas precisas e confiáveis (SAKUNO et al. 2020). O modelo é vantajoso, uma vez que se baseia em valores tabelados de simples determinação, necessita de poucos dados de entrada e, apresenta baixo custo de implementação, fornecendo uma estimativa da perda de solo e dos sedimentos gerados que são depositados ao longo da área de estudo. O EPM é aplicado em escala de bacia hidrográfica e se baseia em fatores como o clima, a topografia, as características do solo, a geologia da área e o uso e ocupação da terra (EBRAHIMZADEH et al. 2018).

A associação do EPM com Sistemas de Informação Geográfica (SIG) permite espacializar as perdas de solo e identificar áreas com maior intensidade de erosão. Além disso, o uso de SIG facilita a execução dos cálculos e proporciona maior confiabilidade aos resultados gerados (SHAHABI et al. 2016).

Nesse contexto, o objetivo do trabalho foi aplicar o Método da Erosão Potencial para estimar as taxas de perdas de solo por erosão hídrica em uma sub-bacia do sul de Minas Gerais e depois elencar as medidas de mitigação dos impactos ambientais, devido ao uso e ocupação do solo, para aliar conservação dos recursos naturais e sustentabilidade socioeconômica e ambiental em sub-bacias hidrográficas.

\section{Materiais e métodos}

\section{1 Área de estudo}

A área de estudo compreende a sub-bacia hidrográfica do Córrego do Pântano II, no Município de Alfenas, sul de Minas Gerais, Sudeste do Brasil (Figura 1). A sub-bacia é um canal de $2^{\mathrm{a}}$ ordem que teve sua foz suprimida com o represamento e criação do reservatório da Usina Hidrelétrica de Furnas em 1963.

$\mathrm{O}$ arcabouço geológico da área é formado por granada-biotita-gnaisses e biotita-gnaisses sobrepostos por coberturas quaternárias de solos, com depósitos fluviais inconsolidados de cascalho, areia e lama (UFRJ e CPRM, 2010). O clima da região, de acordo com a classificação de Köppen, é o Tropical Mesotérmico (Cwb) (SPAROVEK et al. 2007). 


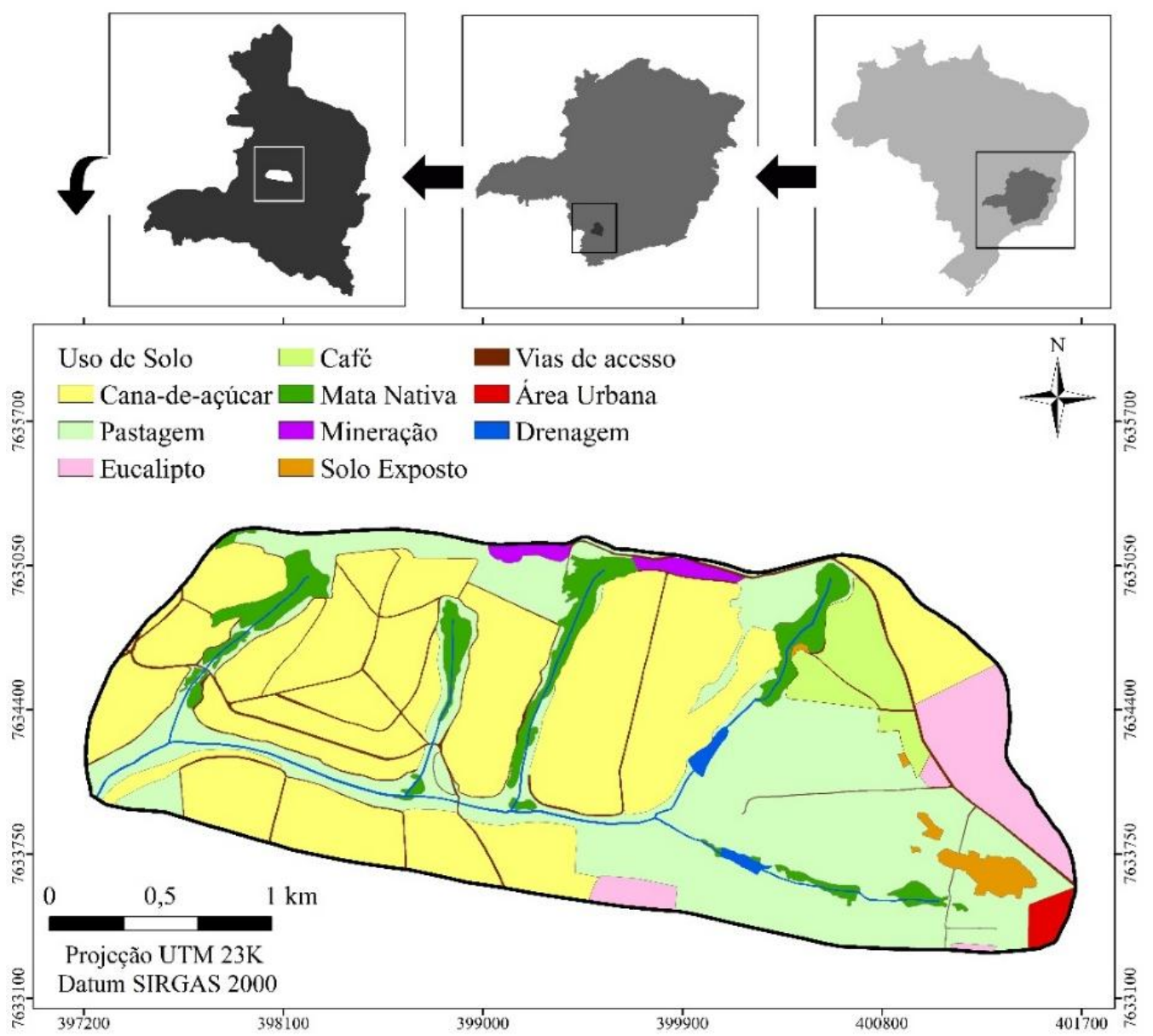

Figura 1: Mapa de localização e de uso e ocupação do solo da Sub-bacia Hidrográfica do Córrego Pântano II, Alfenas, Minas Gerais, Sudeste do Brasil.

Para a confecção do mapa de uso e ocupação da terra (Figura 1) foi realizado o processamento digital de imagens, com fusão das bandas multiespectrais com a banda pancromática aumentando a resolução espacial para $15 \mathrm{~m}$ do sensor OLI acoplado ao satélite Landsat-8, órbita 219, ponto 75, de 14 de agosto de 2018, obtidas da Divisão de Geração de Imagens (INPE, 2019).

As classes de uso e ocupação das terras na sub-bacia são: cana-de-açúcar (287,0 ha), pastagem (214,0 ha), eucalipto (32,3 ha), café ( $21,9 \mathrm{ha})$, mata nativa (34,4 ha), solo exposto $(6,7 \mathrm{ha})$, mineração (5,3 ha), vias de acesso (12,9 ha), área urbana (3,8 ha) e drenagem (5,1 ha).

O mapa digital de solos foi obtido pelo cruzamento do mapa de solos do Estado de Minas Gerais, na escala de 1:650.000 (UFV et al. 2010) com as classes de relevo da área, baseando-se na variação topográfica, como elemento de formação do solo (MCBRATNEY et al. 2003). Desta forma, inicialmente foi obtido o modelo digital de elevação (MDE) (Figura 2A) a partir da interpolação das curvas de nível da Carta Topográfica de Alfenas (FOLHA SF 23-1-1-3) na escala de 1:50.000 (IBGE, 1970), com a ferramenta Topo to Raster no software ARCGIS 10.2 (ESRI, 2015). Utilizando a ferramenta Slope do ARCGIS 10.2 (ESRI, 2015) foi confeccionado o mapa de declividade, com resolução espacial de $10 \mathrm{~m}$ (Figura 2B), representando as classes de relevo (EMBRAPA, 2018).

Os solos foram classificados em Latossolo Vermelho distrófico em relevo plano - LVd1 (declividade 08\%), suave ondulado - LVd2 (declividade 8-20\%), ondulado - LVd3 (declividade 20-45\%) e solos indiscriminados de várzea nas planícies aluviais e zonas de deposição (SIV), correspondendo respectivamente à 49,47\%, 36,49\%, 8,56\% e 5,48\% da área (Figura 2C). 


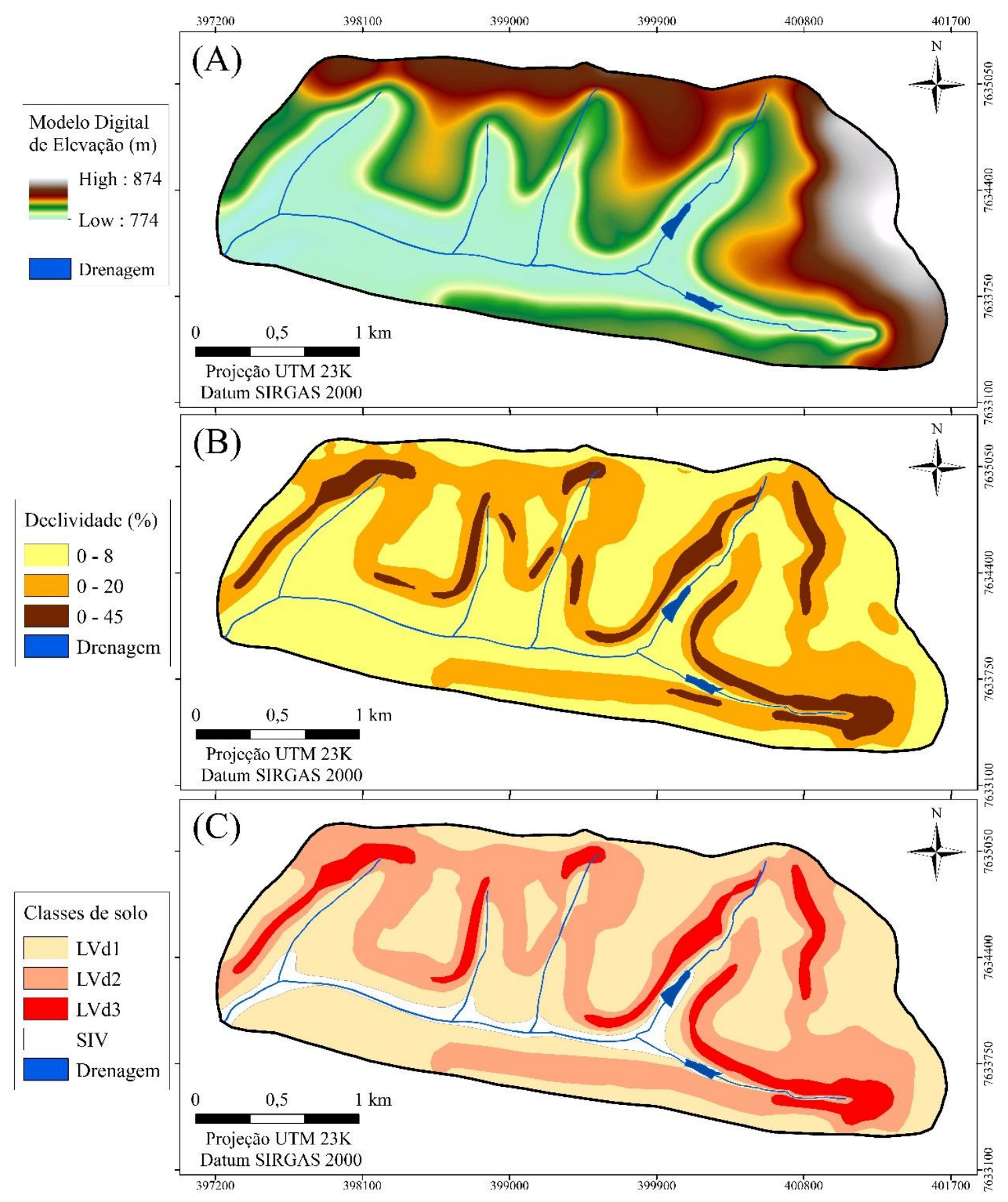

Figura 2: Modelo digital de elevação (MDE) (A); mapa de declividade (B) e mapa de classes de solo (C) da Sub-bacia Hidrográfica do Córrego Pântano II, Alfenas, Minas Gerais, Sudeste do Brasil. Notas: LVd1: Latossolo Vermelho distrófico em relevo plano; LVd2: em relevo suave ondulado; LVd3: em relevo ondulado; SIV: Solos Indiscriminados de Várzea.

\subsection{Método de erosão potencial}

A estimativa de perda de solo foi calculada pelo EPM (GAVRILOVIC, 1988) conforme equações da Tabela 1.

A sub-bacia apresenta uma área (F) de $6,23 \mathrm{~km}^{2}$ e perímetro $(\mathrm{O})$ de $10,94 \mathrm{~km}$. O comprimento, medido a partir dos cursos hídricos (L), é de 4,14 km e a diferença média de elevação (D), calculada a partir da altitude média $(803,64 \mathrm{~m})$ menos a altitude mínima $(773,44 \mathrm{~m})$ é de $30,20 \mathrm{~m}$. Os parâmetros físicos da sub-bacia foram obtidos em SIG, por geoprocessamento com base na Carta Topográfica de Alfenas (IBGE, 1970). 
Tabela 1: Equações e descrições dos parâmetros utilizados para estimativa das perdas de solo no Método de Erosão Pontencial

\begin{tabular}{|c|c|}
\hline & $\mathrm{W}_{\mathrm{yr}}=\operatorname{erosão}$ anual $\left(\mathrm{Mg}\right.$ ano $\left.^{-1}\right)$ \\
\hline & $\mathrm{T}=$ coeficiente de temperatura $\left(\mathrm{Adm}^{(*)}\right)$ \\
\hline \multirow[t]{3}{*}{ (Eq. 1) $\mathrm{W}_{\mathrm{yr}}=\mathrm{T} \cdot \mathrm{H}_{\mathrm{yr}} \cdot \pi \cdot \sqrt[2]{\mathrm{Z}^{3}} \cdot \mathrm{Ds} \cdot \mathrm{F}$} & $\mathrm{H}_{\mathrm{yr}}=$ precipitação anual média $\left(\mathrm{mm} \mathrm{ano}^{-1}\right)$ \\
\hline & $\mathrm{Z}=$ coeficiente de erosão $(\mathrm{Adm})$ \\
\hline & $\mathrm{F}=$ área de estudo $\left(\mathrm{km}^{2}\right)$ \\
\hline \multirow{2}{*}{ (Eq. 2) $\mathrm{G}_{\mathrm{yr}}=\mathrm{W}_{\mathrm{yr}} \cdot \mathrm{R}_{\mathrm{u}}$} & Ds $=$ densidade do solo $\left(\mathrm{kg} \mathrm{dm}^{-3}\right)$ \\
\hline & $\mathrm{G}_{\mathrm{yr}}=$ perda real de solo $\left(\mathrm{Mg}\right.$ ano $\left.^{-1}\right)$ \\
\hline \multirow{3}{*}{ (Eq. 3) $\mathrm{T}=\sqrt[2]{\frac{\mathrm{t}_{0}}{10}}+0,1$} & $\mathrm{R}_{\mathrm{u}}=$ coeficiente de retenção $(\mathrm{Adm})$ \\
\hline & $\mathrm{t}_{0}=$ temperatura média do $\operatorname{ar}\left({ }^{\circ} \mathrm{C}\right.$ ano $\left.{ }^{-1}\right)$ \\
\hline & $\mathrm{Y}=$ coeficiente de resistência a erosão (Adm) \\
\hline \multirow{2}{*}{ (Eq. 4) $\mathrm{Z}=\mathrm{Y} \cdot \mathrm{X}_{\mathrm{a}} \cdot\left(\varphi+\sqrt[2]{\mathrm{I}_{\mathrm{sr}}}\right)$} & $\mathrm{X}_{\mathrm{a}}=$ uso e manejo do solo (Adm) \\
\hline & $\varphi=$ erosão observada em campo (Adm) \\
\hline \multirow{4}{*}{ (Eq. 5) $\mathrm{R}_{\mathrm{u}}=\frac{(\mathrm{O} \cdot \mathrm{D})^{0,5}}{0,25 \cdot(\mathrm{L}+10)}$} & $\mathrm{I}_{\mathrm{sr}}=$ declividade média $(\%)$ \\
\hline & $\mathrm{O}=$ perímetro da sub-bacia $(\mathrm{km})$ \\
\hline & $\mathrm{D}=$ diferença média de elevação $(\mathrm{km})$ \\
\hline & $\mathrm{L}=$ comprimento da sub-bacia $(\mathrm{km})$ \\
\hline
\end{tabular}

Notas: Adm = Adimensional. Fonte: Gavrilovic (1988).

O coeficiente de erosão $(\mathrm{Z})$ representa a intensidade do processo erosivo em determinada área. Quanto mais próximo o valor de $\mathrm{Z}$ estiver de 0 menor será a gravidade da erosão hídrica. $\mathrm{O}$ cálculo de $\mathrm{Z}$ foi feito a partir da declividade média do solo $\left(\mathrm{I}_{\mathrm{sr}}\right)$ e de valores tabelados $\left(\mathrm{Y}, \mathrm{X}_{\mathrm{a}}, \varphi\right)$.

O coeficiente de resistência a erosão (Y) está associado ao material de origem do solo e à geologia da área, com valores variando de 0,25 a 2,0. De acordo com o uso da terra e sua cobertura vegetal foi determinado o coeficiente de uso e manejo do solo $\left(X_{a}\right)$. Valores de $X_{a}$ próximos a zero indicam maior densidade vegetal enquanto valores próximos a 1,0 são característicos de áreas com solo exposto. A erosão observada em campo $(\varphi)$ representa a ocorrência de feições erosivas associadas aos usos do solo. Os valores variam de 0,01 para áreas com erosão laminar fraca e 1,0 para erosão severa (GAVRILOVIC, 1988). Os parâmetros $\mathrm{Y}, \mathrm{X}_{\mathrm{a}}, \varphi$ foram determinados a partir de valores tabelados conforme Sakuno et al. (2020), que adaptaram o EPM para as condições edafoclimáticas tropicais.

A declividade média do solo $\left(\mathrm{I}_{\mathrm{sr}}\right)$ foi determinada a partir do mapa de declividade do solo (Figura 2B) e os parâmetros climáticos $\left(\mathrm{H}_{\mathrm{yr}}, \mathrm{t}_{0}\right)$ obtidos do bancos de dados do INMET (2019).

Os cálculos referentes ao EPM foram realizados no programa IntErO (SPALEVIC, 2011), e a distribuição espacial das perdas de solo foram feitas utilizando o ARCGIS 10.2 (ESRI, 2015) pela ferramenta Raster Calculator.

\section{Resultados e discussões}

A sub-bacia apresenta temperatura média do ar $\left(\mathrm{t}_{0}\right)$ de $22^{\circ} \mathrm{C}$ e a precipitação média anual $\left(\mathrm{H}_{\mathrm{yr}}\right)$ de 1.500 mm (ALVARES et al. 2013; INMET, 2019; TAVARES et al. 2019). Com base nesses valores o coeficiente de temperatura (T) foi calculado em 1,52. O coeficiente de resistência do solo (Y) foi de 0,90, indicando baixa suscetibilidade do solo à erosão hídrica, bem como a boa permeabilidade, condizente com as características físicas dos Latossolos Vermelhos distróficos e do arcabouço geológico da área. Segundo Tavares et al. (2019), a densidade média do Latossolos na região é de $1,15 \mathrm{~kg} \mathrm{dm}^{-3}$.

$\mathrm{O}$ coeficiente de uso e manejo $\left(\mathrm{X}_{\mathrm{a}}\right)$ foi de 0,54 indicando boas práticas conservacionistas nas áreas cultivadas, com destaque para o plantio em nível da cana-de-açúcar, que ocupa a maior parcela de uso do solo $(46,0 \%)$.

O grau das feições erosivas $(\varphi)$ foi de 0,40 , que demostra a predominância de erosão laminar média (GAVRILOVIC, 1988). Nas áreas de mineração e com solo exposto foi verificada maior severidade das feições erosivas, devido à ausência de cobertura do solo. A declividade média da área é de $8,77 \%$ indicando predomínio de relevo suave ondulado (Figura 2B). O relevo é fator decisivo no processo erosivo já que regula a velocidade e o volume do escoamento superficial e, consequentemente, a quantidade de solo e água perdidos por erosão hídrica (SILVA et al. 2011). 
O coeficiente de intensidade de erosão $(Z)$ variou de 0,1 a 1,2 com média de 0,347 . Segundo a classificação de Gavrilovic (1988), na sub-bacia ocorre predomínio de erosão hídrica de fraca intensidade. Nas áreas com cana-de-açúcar a média de $\mathrm{Z}$ foi 0,540, que indica erosão moderada (Figura 3A).

A estimativa de erosão total $\left(\mathrm{W}_{\mathrm{yr}}\right)$ foi de $10.080,00 \mathrm{Mg}$ ano $^{-1}$. O coeficiente de retenção $\left(\mathrm{R}_{\mathrm{u}}\right)$ foi calculado em 0,081 , indicando que $8,10 \%$ dos sedimentos gerados atingem os corpos hídricos, contribuindo para o assoreamento do reservatório da Usina Hidrelétrica de Furnas. Desta forma, considerando o $R_{u}$, a perda de solo real $\left(\mathrm{G}_{\mathrm{yr}}\right)$ foi de $816,48 \mathrm{Mg}$ noo $^{-1}$ com perda média estimada em 1,31 $\mathrm{Mg} \mathrm{ha}^{-1}$ ano $^{-1}$ (Figura 3B).

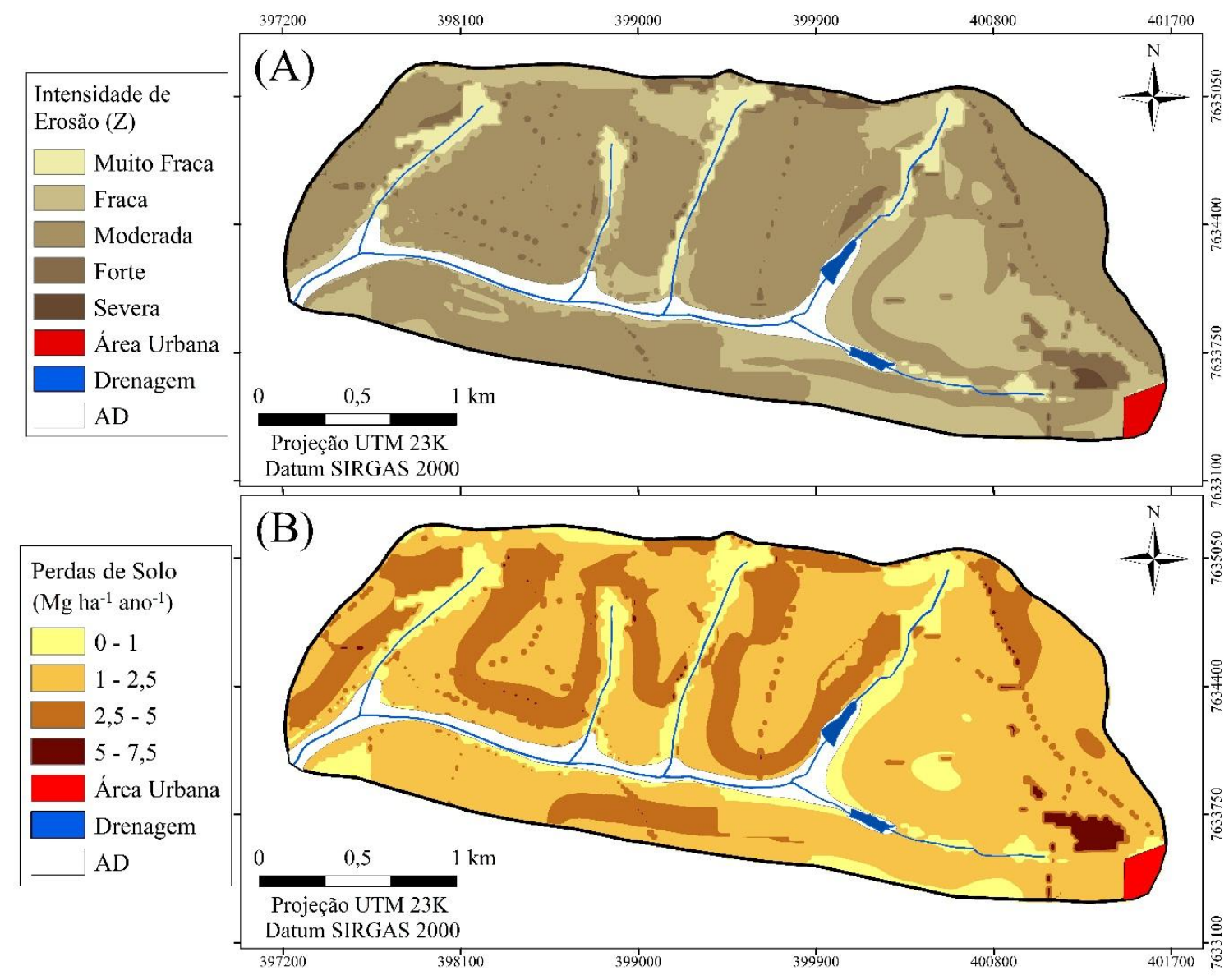

Figura 3: Mapa do coeficiente de intensidade de Erosão (Z) (A) e mapa de perdas de solo (B) na sub-bacia Hidrográfica do Córrego Pântano II, Alfenas, Minas Gerais, Sudeste do Brasil. Notas: AD: área de deposição.

Nos cálculos das perdas de solos, as áreas ocupadas por SIV (Figura 2C) não foram consideradas, pois se caracterizam como áreas de deposição e acúmulo de sedimentos (AYER et al. 2015).

De acordo com o esperado, a mata nativa apresentou a menor taxa de perda de solo, com valor estimado em $0,17 \mathrm{Mg} \mathrm{ha}^{-1}$ ano $^{-1}$, devido à densa cobertura vegetal dessa classe de uso. Nas áreas de cana-de-açúcar, eucalipto, pastagem e café, as quais apresentavam práticas conservacionistas como plantio em nível, manutenção da vegetação espontânea e dos restos vegetais, foram obtidos baixos níveis de perdas (Tabela 2). Para a cana-de-açúcar, a perda média de solo $\left(1,74 \mathrm{Mg} \mathrm{ha}^{-1}\right.$ ano $\left.^{-1}\right)$ foi próxima à encontrada por Youlton et al. (2016), que avaliaram as perdas de solo em canaviais do Estado de São Paulo por meio de parcelas experimentais. Este cultivo apresentou a maior contribuição nas perdas totais na área, por ocupar a maior área de uso do solo (Tabela 2). No caso, o plantio direto é uma excelente alternativa para o cultivo da canade-açúcar. É uma prática que pode contribuir para diminuir a erosão hídrica, pelo uso dos restos vegetais do cultivo anterior no novo plantio, aumentando a proteção do solo e incorporando matéria orgânica que melhora a estrutura do solo (BERTONI e LOMBARDI NETO, 2012).

Nas áreas de solo exposto, mineração e nas vias de acesso, conforme as expectativas, foram verificadas as maiores taxas de erosão, com perdas de solo de 3,92, 2,86 e 2,01 $\mathrm{Mg} \mathrm{ha}^{-1}$ ano $^{-1}$, respectivamente. Nessas classes de uso, a maior produção de sedimentos está associada a ausência de cobertura vegetal evidenciando o papel das práticas vegetativas na conservação do solo. 
Tabela 2: Perdas de solo estimadas pelo Método de Erosão Potencial (EPM) para as classes de uso de terra na sub-bacia Hidrográfica do Córrego Pântano II, Alfenas, Minas Gerais, Sudeste do Brasil.

\begin{tabular}{cccc}
\hline Uso da Terra & Área $(\mathbf{h a})$ & Perda Média $\left(\mathbf{M g ~ h a}^{\mathbf{- 1}} \mathbf{a n o}^{\mathbf{- 1}}\right)$ & Perda Total $(\mathbf{M g}$ ano \\
\hline Cana-de-açúcar & 287,0 & 1,74 & 499,38 \\
Pastagem & 179,9 & 0,90 & 161,91 \\
Eucalipto & 32,3 & 1,63 & 52,65 \\
Café & 21,9 & 1,34 & 29,35 \\
Mata Nativa & 34,4 & 0,17 & 5,85 \\
Solo Exposto & 6,7 & 3,92 & 26,26 \\
Mineração & 5,3 & 2,86 & 15,15 \\
Vias de acesso & 12,9 & 2,01 & 25,93 \\
Área Urbana* & 3,8 & - & - \\
Área de Deposição** & 34,1 & - & - \\
Drenagem* & 5,3 & - & - \\
\hline Total & 623,6 & - & 816,48 \\
\hline
\end{tabular}

*Áreas não consideradas no cálculo de perdas de solo. **Área de deposição: calculada com medições em campo, coberta principalmente com pastagem.

Uma maneira de avaliar se a taxa de erosão está em níveis aceitáveis é utilizando os valores do limite de Tolerância de Perda de Solo (TPS) como parâmetro de comparação. A TPS é um parâmetro que indica o valor máximo da taxa de erosão que ainda permitirá uma produção agrícola ser sustentável (WISCHMEIER e SMITH, 1978). Segundo Mendes Júnior et al. (2018), a TPS para os Latossolos Vermelhos distróficos de Alfenas, Minas Gerais, variam de acordo com o relevo em 5,19 a 5,90 $\mathrm{Mg} \mathrm{ha}^{-1} \mathrm{ano}^{-1}$. Considerando esses valores, cerca de $1,0 \%$ da sub-bacia apresentou perdas acima dos limites da TPS. As maiores perdas se concentraram principalmente nas áreas de solo exposto e nas vias de acesso com declividades íngremes. Essas áreas devem ser prioritárias para adoção de práticas conservacionistas, buscando reduzir o processo de degradação do solo. Nos locais com perdas abaixo dos limites de TPS, as práticas conservacionistas devem ser ampliadas buscando garantir a sustentabilidade do solo a longo prazo.

Nas áreas com solo exposto, a implementação de forrageiras ou até mesmo o manejo da vegetação espontânea é uma alternativa que pode ser utilizada para reduzir o escoamento superficial. Segundo Dechen et al. (2015), a cobertura do solo influencia diretamente a geração de sedimentos e o aumento da vegetação favorece a agregação das partículas do solo e a retenção de água na unidade produtiva. Para as vias de acesso, práticas como a adição de cascalho e a construção de bacias de contenção, principalmente em relevos acentuados, podem ajudar a reduzir as perdas de solo, uma vez que em estradas não pavimentadas, o processo erosivo é significativamente superior (OLIVEIRA et al. 2010).

As estimativas geradas pelo EPM apresentaram valores próximos daqueles encontrados por outros autores na mesma região, que utilizaram modelos tradicionais e adaptados às condições tropicais, como a RUSLE e o próprio EPM, indicando confiabilidade dos resultados obtidos (MENDES JÚNIOR et al. 2018; TAVARES et al. 2019). Além disso, de maneira geral, os modelos preditivos de erosão são mais eficientes em apontar as áreas com elevadas perdas de solo, uma vez que nessas condições os erros associados aos métodos são menores (AMORIM et al. 2010). Esse é um aspecto de elevada relevância, já que são justamente essas áreas que devem ser prioritariamente identificadas, para que medidas mitigadoras sejam planejadas e implementadas.

Os resultados obtidos permitem sugerir medidas para reduzir as perdas de solos na agricultura. Dentre elas, o plantio direto e em curvas de nível, adubação orgânica e associação de diferentes cultivos em sistemas agroflorestais de produção. Tais técnicas permitem a redução das perdas de solo pela erosão hídrica e de nutrientes pela lixiviação, preservando sua capacidade produtiva. Em cultivos temporários, como o da canade-açúcar, os manejos conservacionistas são fundamentais para manter as perdas de solo em níveis abaixo da TPS, pois no período entre safras é comum o solo ficar exposto, aumentando o potencial deletério da ação erosiva (AYER et al. 2015).

Estudos referentes a modelagem da erosão hídrica subsidiam a definição de áreas prioritárias de atuação para contenção das perdas de solo. Tais estudos em conjunto com as classes de capacidade de uso da terra (LEPSCH et al. 2015), aplicado em sub-bacias hidrográficas do sul de Minas Gerais, permitiram elaborar planos de manejo eficazes para reduzir as perdas de solo nas atividades agropecuárias. Além disso, é possível adotar medidas que visem assegurar a prestação de serviços ambientais e ecossistêmicos, como a manutenção do equilíbrio hidrosedimentológico de bacias hidrográficas e a manutenção da qualidade das águas e dos solos (SERVIDONI et al. 2016). 
As perdas de solos, conforme Ayer et al. (2015), Mendes Junior et al. (2018) e Lense et al. (2019), podem comprometer a capacidade produtiva dos solos. Assim, podem impactar diretamente a produção de alimentos e comprometer a segurança alimentar e nutricional da população. Dessa forma, a conservação do solo no processo produtivo agrícola é fundamental para manter a população em segurança. Pois, a pressão sobre os solos devido ao crescimento da demanda por alimentos tem aumentado e as projeções demonstram que tal demanda será ainda maior nas próximas décadas. Dessa forma, propostas de modelagem da erosão hídrica aliadas ao plano de manejo para conservação dos solos e da água são necessárias para alcançar a sustentabilidade agrícola (SERVIDONI et al. 2019).

\section{Conclusões}

A perda de solo média estimada para a área de estudo foi de $1,31 \mathrm{Mg} \mathrm{ha}^{-1}$ ano $^{-1}$, variando de acordo com as classes de uso do solo de 0,17 a 3,92 $\mathrm{Mg} \mathrm{ha}^{-1}$ ano $^{-1}$.

O Método de Erosão Potencial é uma alternativa eficaz, simples e de baixo custo para identificar áreas prioritárias para ações de mitigação dos impactos ambientais associados à erosão hídrica. As estimativas fornecidas pelo método podem contribuir para o planejamento do uso conservacionista do solo a longo prazo.

As estimativas das taxas de perdas de solo por erosão hídrica podem ser ferramentas auxiliares essenciais para definição das medidas mitigadoras e, consequentemente, para contribuir com a manutenção da capacidade produtiva dos solos e com a prestação de serviços ambientais e ecossistêmicos e a soberania e segurança alimentar e nutricional da população.

\section{Referências}

ALVARES, C.A.; STAPE, J.L.; SENTELHAS, P.C.; GONÇALVES, J.L.M.; SPAROVEK, G. Köppen's climate classification map for Brazil. Meteorologische Zeitschrift, Stuttgart, v. 22, p. 711-728, 2013. https://doi.org/10.1127/0941-2948/2013/0507

AMORIM, R.S.S.; SILVA, D.D.; PRUSKI, F.F.; MATOS, A.T. Avaliação do desempenho dos modelos de predição da erosão hídrica USLE, RUSLE e WEPP para diferentes condições edafoclimáticas do Brasil. Engenharia Agrícola, Jaboticabal, v. 30, p. 1046-1049, 2010. https://doi.org/10.1590/S010069162010000600006

AVANZI, J.C.; SILVA, M.L.N.; CURI, N.; NORTON, L.D.; BESKOW, S.; MARTINS, S.G. Spatial distribution of water erosion risk in a watershed with eucalyptus and Atlantic Forest. Ciência e Agrotecnologia, Lavras, v. 37, n. 5, p. 427-434, 2013. https://doi.org/10.1590/S1413-70542013000500006

AYER, J.E.B.; OLIVETTI, D.; MINCATO, R.L.; SILVA, M.L.N. Erosão Hídrica em Latossolos Vermelhos Distróficos. Pesquisa Agropecuária Tropical, Goiânia, v. 45, p. 180-191, 2015. https://doi.org/10.1590/1983-40632015v4531197

BARROS, E.N.S.; VIOLA, M.R.; RODRIGUES, J.A.M.; MELLO, C.R.; AVANZI, J. C.; GIONGO, M. Modelagem da erosão hídrica nas bacias hidrográficas dos rios Lontra e Manoel Alves Pequeno, Tocantins. Revista Brasileira de Ciências Agrárias, Recife, v. 13, p. 1-9, 2018. htps://doi.org/10.5039/agraria.v13i1a5509

BERTONI J.; LOMBARDI NETO F. Conservação do solo. 8. ed. São Paulo: Ícone Editora; 2012. 355 p.

DECHEN, S.C.F.; TELLES, T.S.; GUIMARAES, M.F.; MARIA, I.C. Perdas e custos associados à erosão hídrica em função de taxas de cobertura do solo. Bragantia, Campinas, v. 74, p. 224-233, 2015. https://doi.org/10.1590/1678-4499.0363

EBRAHIMZADEH, S.; MOTAGH, M.; MAHBOUB, V.; HARIJANI, F.M. An improved RUSLE/SDR model for the evaluation of soil erosion. Environmental Earth Sciences, Amsterdam, v. 77, p. 1-17, 2018. https://doi.org/10.1007/s12665-018-7635-8.

EMBRAPA - Empresa Brasileira de Pesquisa Agropecuária. Sistema Brasileiro de Classificação de Solos. 5. ed., rev. e ampl. Brasília, DF: Embrapa, 2018. 356 p. 
ESRI, Environmental Systems Research Institute - Inc. ARCGIS Professional GIS for the desktop version 10.3. Redlands, California, EUA, Software, 2015.

FAO - FOOD AND AGRICULTURE ORGANIZATION OF THE UNITED NATIONS. Global Soil Partnership Endorses Guidelines on Sustainable Soil Management, 2017. Disponível em: <http://www.fao.org/global-soil-partnership/resources/highlights/detail/en/c/416516/>

GAVRILOVIC, S. The use of empirical method (erosion potential method) for calculating sediment production and transportation in unstudied or torrential streams. In: WHITE, W.R. (Ed.) International Conference on River Regime, Chichester, 1988. p. 411-422.

HERNANI, L.C.; FREITAS, P.L.; DENARDIN, J.E.; KOCHHAM, R.A.; DE-MARIA, I.C.; LANDERS, J.N. A erosão e seu impacto. In: MANZATTO, C.V.; FREITAS JÚNIOR, E.; PERES, J.R.R (Org.) Uso agrícola dos solos brasileiros. Rio de Janeiro: Embrapa Solos, 2002, p. 47-60.

IBGE - Instituto Brasileiro de Geografia e Estatística. Carta Topográfica do Município de Alfenas. (FOLHA SF 23-1-1-3). Escala 1:50.000. Rio de Janeiro: IBGE, 1970.

INMET - Instituto Nacional de Meteorologia. Estações pluviométricas convencionais, 2018. Disponível em: <http://www.inmet.gov.br/portal/index.php?r=bdmep/bdmep>.

INPE - Instituto Nacional de Pesquisas Espaciais. SGI 2.5 Divisão de Geração de Imagens (DIDGI). SGI. Imagem Geossistemas, São José dos Campos: Instituto Nacional de Pesquisas Espaciais, 2019.

LENSE, G.H.E.; CRISTO, T.; MOREIRA, R.S.; AVANZI, J.C.; MINCATO, R.L. Estimates of soil losses by the erosion potential method in tropical latosols. Ciência e Agrotecnologia, Lavras, v.43, p. 1-10, 2019. https://doi.org/10.1590/1413-7054201943012719

LEPSCH, I.F.; ESPINDOLA, C.R.; VISCHI FILHO, W.J.; HERNANI, L.C.; SIQUEIRA, D.S. Manual para levantamento utilitário e classificação de terras no sistema de capacidade de uso. 1. ed. Viçosa: Sociedade Brasileira de Ciência do Solo; 2015. 175 p.

LOVRIC, N.; TOSIC, R. Assessment of soil erosion and sediment yield using erosion potential method: Case study - Vrbas river basin (B\&H). Bulletin of the Serbian Geographical Society, Belgrade, v. 98, p. 114, 2018. https://doi.org/10.2298/GSGD180215002L

MCBRATNEY, A.B.; SANTOS, M.L.M.; MINASNY, B. On digital soil mapping. Geoderma, Amsterdam, v. 117, p. 3-52, 2003. https://doi.org/10.1016/S0016-7061(03)00223-4

MENDES JÚNIOR, H.; TAVARES, A.S.; SANTOS JÚNIOR, W.R.; SILVA, M.L.N.; SANTOS, B.R.; MINCATO, R.L. Water Erosion in Oxisols under Coffee Cultivation. Revista Brasileira de Ciência do Solo, Viçosa, v. 42, p. 1-14, 2018. https://doi.org/10.1590/18069657rbcs20170093

MERTEN, G.H.; MINELLA, J.P.G. The expansion of Brazilian agriculture: Soil erosion scenarios. International Soil and Water Conservation Research, Amsterdam, v. 1, p. 37-48, 2013. https://doi.org/10.1016/S2095-6339(15)30029-0

OLIVEIRA, F.P.; SILVA, M.L.N.; AVANZI, J.C.; CURI, N.; LEITE, F.P. Avaliação de perdas de solo em estradas florestais não pavimentadas no Vale do Rio Doce, Minas Gerais. Scientia Forestalis, Piracicaba, v. 38, p. 331-339, 2010. Disponível em: 〈http:Ilwww.ipef.br>.

PANAGOS, P.; STANDARDI, G.; BORRELLI, P.; LUGATO, E.; MONTANARELLA, L.; BOSELLO, F. Cost of agricultural productivity loss due to soil erosion in the European Union: From direct cost evaluation approaches to the use of macroeconomic models. Land Degradation \& Development, London, v. 29, p. 471-484, 2018. https://doi.org/10.1002/1dr.2879 
PANDEY, A.; HIMANSHU, S.K.; MISHRA, S.K.; SINGH, V.P. Physically based soil erosion and sediment yield models revisited. Catena, Amsterdam, v. 147, p. 595-620, 2016. https://doi.org/10.1016/j.catena.2016.08.002

POSTHUMUS, H.; DEEKS, L.K.; RICKSON, R.J.; QUINTON, J.N. Costs and benefits of erosion control measures in the UK. Soil Use and Management, London, v. 31, p. 16-33, 2015. https://doi.org/10.1111/sum.12057

SAKUNO, N.R.R.; GUIÇARDI, A.C.F.; SPALEVIC, V.; SILVA, M.L.N.; MINCATO, R.L. Adaptation and application of the erosion potential method for tropical soils. Revista Ciência Agronômica, Fortaleza, v. 51, p. 1-10, 2020. https://doi.org/10.5935/1806-6690.20200004

SERVIDONI, L.E.; AYER, J.E.B.; SILVA, M.L.N.; SPALEVIC, V.; MINCATO, R.L. Land use capacity and environment services. Revista Brasileira de Geografia Física, Recife, v. 09, p. 1712-1724, 2016. https://doi.org/10.26848/rbgf.v9.6.p1712-1724

SERVIDONI, L.E.; OLIVEIRA JÚNIOR, B.M.; BISSOLI, M.C.; MINCATO, R.L. Capacidade de uso das terras, conservação do solo e produção agrícola: estudo de caso da sub-bacia hidrográfica do Ribeirão Caçús, Alfenas - MG. Interespaço: Revista de Geografia e Interdisciplinaridade, Grajaú, v. 05, p. 01-20, 2019. https://doi.org/10.18764/2446-6549.2019.12119

SHAHABI, H.; MAMAND SALARI, M.; AHMAD, B. B., MOHAMMADI, A. Soil erosion hazard mapping in Central Zab Basin using EPM model in GIS environment. International Journal of Geography and Geology, Islamabad, v. 5, p. 224-235, 2016. https://doi.org/10.18488/journal.10/2016.5.11/10.11.224.235

SCHARRÓN, C.E.R.; SÁNCHEZ, Y.F. Plot-, Farm-, and Watershed-Scale Effects of Coffee Cultivation in Runoff and Sediment Production in Western Puerto Rico. Journal of Environmental Management, Amsterdam, v.202, n.1, p. 126-136, 2017. https://doi.org/10.1016/j.jenvman.2017.07.020

SILVA, M.A.; SILVA, M.L.N.; CURI, N.; AVANZI, J.C.; LEITE, F.P. Sistemas de manejo em plantios florestais de eucalipto e perdas de solo e água na região do Vale do Rio Doce, MG. Ciência Florestal, Santa Maria, v. 21, p. 765-776, 2011. https://doi.org/10.5902/198050984520

SPALEVIC, V. IntErO, Intensity of Erosion and Outflow [internet]. Version 1.0. Podgorica: Univerzitet Crne Gore, Software, 2011. Disponível em: <www.agricultforest.ac.me/Spalevic/IntErO>.

SPAROVEK, G.; VAN LIER, Q. J.; DOURADO NETO, D.D. Computer assisted Koeppen climate classification: a case study for Brazil. International Journal of Climatology, London, v. 27, p. 257-266, 2007. https://doi.org/10.1002/joc.1384.

TAVARES, A.S.; SPALEVIC, V.; AVANZI, J.C.; NOGUEIRA, D.A.; SILVA, M.L. N.; MINCATO, R.L. Modeling of water erosion by the erosion potential method in a pilot subbasin in southern Minas Gerais. Semina: Ciências Agrárias, Londrina, v. 40, p. 555-572, 2019. https://doi.org/10.5433/16790359.2019v40n2p555

UFRJ, Universidade Federal do Rio de Janeiro. CPRM, Companhia de Pesquisa de Recursos Minerais. Mapa Geológico da Folha Alfenas (SF-23-V-D-II). Escala 1:100.000. Rio de Janeiro: CPRM, 2010.

UFV; CETEC; UFLA; FEAM. Mapa de Solos do Estado de Minas Gerais. Escala 1:650:000. Belo Horizonte: Fundação Estadual do Meio Ambiente, 2010. 49 p.

WISCHMEIER, W.H; SMITH, D.D. Predicting rainfall erosion losses: a guide to conservation planning. Washington: United States Department of Agriculture. Supersedes Agriculture Handbook. 1978. 67 p.

YOULTON, C.; WENDLAND, E.; ANACHE, J.A.A.; POBLETE-ECHEVERRIA, C.; DABNEU, S. Changes in erosion and runoff due to replacement of pasture land with sugarcane crops. Sustainability, Basel, v.8, p. 1-12, 2016. https://doi.org/10.3390/su8070685 